\title{
Numerical Techniques in Lévy Fluctuation Theory
}

\author{
Naser M. Asghari • Peter den Iseger • Michael Mandjes
}

Received: 19 December 2011 / Revised: 24 May 2012 /

Accepted: 20 June 2012 / Published online: 24 August 2012

(C) The Author(s) 2012. This article is published with open access at Springerlink.com

\begin{abstract}
This paper presents a framework for numerical computations in fluctuation theory for Lévy processes. More specifically, with $\bar{X}_{t}:=\sup _{0 \leq s \leq t} X_{s}$ denoting the running maximum of the Lévy process $X_{t}$, the aim is to evaluate $\mathbb{P}\left(\bar{X}_{t} \leq x\right)$ for $t, x>$ 0 . We do so by approximating the Lévy process under consideration by another Lévy process for which the double transform $\mathbb{E} e^{-\alpha \bar{X}_{\tau(q)}}$ is known, with $\tau(q)$ an exponentially distributed random variable with mean $1 / q$; then we use a fast and highly accurate Laplace inversion technique (of almost machine precision) to obtain the distribution of $\bar{X}_{t}$. A broad range of examples illustrates the attractive features of our approach.
\end{abstract}

Keywords Lévy processes • Fluctuation theory • Wiener-Hopf • Phase-type distributions $\cdot$ Mathematical finance

AMS 2000 Subject Classifications $60 \mathrm{G} 51 \cdot 65 \mathrm{~T} 99$

\footnotetext{
N. M. Asghari · M. Mandjes ( $\varangle)$

Korteweg-de Vries Institute for Mathematics, University of Amsterdam, Amsterdam, the Netherlands e-mail: M.R.H.Mandjes@uva.nl

N. M. Asghari

e-mail: n.mohammadasghari@uva.nl

P. den Iseger

ABN-Amro, Amsterdam, the Netherlands

e-mail: peter.den.iseger@nl.abnamro.com

M. Mandjes

Eurandom, Eindhoven University of Technology, Eindhoven, the Netherlands

M. Mandjes

CWI, Amsterdam, the Netherlands
} 


\section{Introduction}

Owing to their wide applicability and their attractive mathematical properties, Lévy processes play an important role in applied probability. In mathematical terms, they are characterized as processes with stationary and independent increments, and, as such, the class of Lévy processes covers e.g. Brownian motion and (compound) Poisson processes (but is substantially broader; for instance processes with infinitely many jumps in finite time intervals belong to this class as well). Over the past decades Lévy processes have found widespread use in various application domains. More specifically, they are intensively studied in both mathematical finance and operations research, see, among many other sources, for instance Asmussen (2003), Cont and Tankov (2004) and Dȩbicki and Mandjes (2012).

With $X_{t}$ denoting the Lévy process (assuming $X_{0}=0$ ), a substantial research effort concentrates on analyzing probabilistic properties of the so-called running maximum process $\bar{X}_{t}:=\sup _{0 \leq s \leq t} X_{s}$. More particularly, one wishes to determine the probability $\mathbb{P}\left(\bar{X}_{t} \leq x\right)$ for $t, x>0$, or alternatively the corresponding density. The branch of research focusing on this type of problems is commonly known as fluctuation theory (Bertoin 1998; Kyprianou 2006; Prabhu 1998).

A Lévy process is characterized by its Lévy exponent $\log \mathbb{E} e^{\mathrm{i} s X_{1}}$, which is a necessarily of the form

$$
\log \mathbb{E} e^{\mathrm{i} s X_{1}}=\mathrm{i} s d-\frac{1}{2} s^{2} \sigma^{2}+\int_{-\infty}^{\infty}\left(e^{\mathrm{i} s x}-1-\mathrm{i} s x 1_{\{|x|<1\}}\right) \Pi(\mathrm{d} x),
$$

where $d \in \mathbb{R}, \sigma \geq 0$, and the spectral measure $\Pi(\cdot)$, concentrated on $\mathbb{R} \backslash\{0\}$, satisfies

$$
\int_{\mathbb{R}} \min \left\{x^{2}, 1\right\} \Pi(\mathrm{d} x)<\infty .
$$

The triplet $\left(d, \sigma^{2}, \Pi\right)$ is usually referred to as the characteristic triplet, as it uniquely defines the Lévy process (Bertoin 1998, Ch. I, Thm. 1). The three terms in the right-hand side of the representation (1) are, for obvious reasons, often called the (deterministic) drift term, the Brownian term, and the jump term. Special cases of Lévy processes are deterministic drifts (only a drift term) and Brownian motions (only a Brownian term). The class of Lévy processes also contains compound Poisson processes; then we just have the jump-term (and the first term as well in case a deterministic drift is present as well); in addition there should be a well-defined arrival rate (which requires that $\left.\int_{-\infty}^{\infty} \Pi(\mathrm{d} x)<\infty\right)$. The class is wider though, as it also includes processes with infinitely many jumps in a finite amount of time (usually referred to as 'small jumps'); this happens in case $\int_{-\infty}^{\infty} \Pi(\mathrm{d} x)=\infty$.

In principle the distribution of $\bar{X}_{t}$ is fully specified through the so-called WienerHopf decomposition, see e.g. Kyprianou (2006, Ch. 6). It states that, with $\tau(q)$ denoting an exponential random variable with mean $1 / q$ that is independent of the Lévy process $X_{t}$,

$$
\kappa(\alpha, q):=\mathbb{E} e^{-\alpha \bar{X}_{\tau(q)}}=k_{0} \exp \left(-\int_{0}^{\infty} \int_{(0, \infty)} \frac{1}{t}\left(e^{-t}-e^{-q t-\alpha x}\right) \mathbb{P}\left(X_{t} \in \mathrm{d} x\right) \mathrm{d} t\right),
$$


where $k_{0}$ is a normalizing constant. From a practical standpoint, the use of this characterization is limited, as it provides us with the double transform of $\mathbb{P}\left(\bar{X}_{t} \in\right.$ $\mathrm{d} x$ ) -realize that

$$
\frac{1}{q} \cdot \mathbb{E} e^{-\alpha \bar{X}_{\tau(q)}}=\int_{0}^{\infty} e^{-q t} \int_{0}^{\infty} e^{-\alpha x} \mathbb{P}\left(\bar{X}_{t} \in \mathrm{d} x\right) \mathrm{d} t,
$$

which in general cannot be inverted explicitly.

The above entails that, in order to get numerical values for the density $\mathbb{P}\left(\bar{X}_{t} \in \mathrm{d} x\right)$ or the distribution function $\mathbb{P}\left(\bar{X}_{t} \leq x\right)$, one option is to (i) first evaluate the double integral (2) numerically, and then to (ii) numerically invert the double transform (3). The primary objective of this paper is to develop a methodology to evaluate $\mathbb{P}\left(\bar{X}_{t} \leq x\right)$, but to do so by bypassing stage (i) above. The underlying idea is that we make use of the fact that for quite a substantial class of Lévy processes $X_{t}$, the double transform $\kappa(\alpha, q)$ can be expressed explicitly in terms of the Lévy exponent; we replace the Lévy process under consideration by a (suitably chosen) Lévy process in this class, so that the just performing stage (ii) remains.

As mentioned above, for a broad class of Lévy processes the double transform $\kappa(\alpha, q)$ can be expressed explicitly in terms of the Lévy exponent; in some cases still a number of (relatively straightforward) numerical computations need to be performed. We give a brief overview of such processes here.

- The most standard examples in which this is possible are the ones in which the underlying Lévy process is spectrally one-sided. This means that $X_{t}$ has either only negative jumps (the spectrally negative case; write $X \in \mathscr{S}_{-}$) or only positive jumps (the spectrally positive case; write $X \in \mathscr{S}_{+}$). In the former case the running maximum up to the exponential epoch $\tau(q)$ has an exponential distribution, whereas in the latter case the so-called generalized Pollaczek-Khinchine formula applies; see e.g. Dȩbicki and Mandjes (2012, Ch. III and IV). In both cases, $\kappa(\alpha, q)$ can be expressed in closed-form in terms of the Lévy exponent.

- It has been found out more recently that $\kappa(\alpha, q)$ can be expressed in semi-explicit terms if the jumps in one direction (either upward or downward) are phase-type (or, more generally, have a rational Laplace transform), whereas the jumps in the other direction are allowed to have a general distribution-see for results along these lines (Asmussen et al. 2004; Lewis and Mordecki 2005, 2008). In this paper, we concentrate on the setting of Lewis and Mordecki (2005) in which the positive jumps have a rational Laplace transform, and the downward jumps are general; we write $X \in \mathscr{R}$. In this case $\kappa(\alpha, q)$ can be expressed in terms of the zeros of a specific equation (that needs to be solved numerically).

- If the Lévy exponent is a meromorphic function (write: $X \in \mathscr{M}$ ), expressed in terms of beta and digamma functions, the Wiener-Hopf factorization can be done in essentially the same way as in case of phase-type distributed jumps (Kuznetsov 2010; Kuznetsov et al. 2011). This Wiener-Hopf factorization, however, is now in terms of an infinite product, due to the infinitely many poles of the Lévy exponent, so there is a truncation error. In the context of the present paper we consider the class of Beta processes (Kuznetsov 2010, Section 4), which has meromorphic Lévy exponent. 
As indicated above, in our numerical evaluation scheme we approximate the Lévy process under consideration by one in the class for which we can compute the double transform $\kappa(\alpha, q)$ explicitly (that is, a Lévy process in $\mathscr{S}_{-}, \mathscr{S}_{+}$, or $\mathscr{R}$ ). In case there are non-phase-type jumps in both directions, the jumps in one direction are approximated by using a phase-type distribution; if there are 'small jumps', we approximate the jumps of the Lévy process by the sum of an appropriately chosen compound Poisson process and Brownian motion (Asmussen and Rosiński 2004). Then we have an approximation for $\kappa(\alpha, q)$, which is inverted using the inversion approach presented in den Iseger (2006); this approach can be considered as 'state-of-the-art' in terms of accuracy (near machine precision), speed and general applicability.

To the best of our knowledge, our study is the first systematic account that tackles the numerical evaluation of $\mathbb{P}\left(\bar{X}_{t} \leq x\right)$ for $t, x>0$ (or the corresponding density) in full generality. Building on the ideas mentioned above, we study in great detail the numerical accuracy and complexity of our approximation method. This is done for an extensive set of examples, covering many of the specific Lévy processes proposed in the literature. It is noted that particular Lévy processes were already dealt with before, see for instance Asmussen et al. (2007) for the CGMY process; Rogers (2000) and Surya (2008) focus on numerical aspects related to the spectrally-negative case.

The remainder of our paper is organized as follows. Section 2 sketches the preliminaries of our approach: it reviews the results for the spectrally one-sided case as well as the results from Lewis and Mordecki (2005) for the case the positive jumps have a rational Laplace transform. In Section 3 the case of one-sided jumps is dealt with, with a focus on Brownian motion and compound Poisson; the output of the numerical experiments is validated against either exact results or simulationbased results. Then Section 4 studies the effect of replacing the positive jumps by a phase-type counterpart; to assess the accuracy of the method we also perform these approximations for instances that do allow explicit calculation of the double transform $\kappa(\alpha, q)$. Section 5 concerns the approximation of small jumps by the sum of a Brownian motion and a compound Poisson process. When the Wiener-Hopf factorization is available, there is an efficient method (Kuznetsov et al. 2011) for sampling the running maximum (called Wiener-Hopf Monte Carlo, or WH-MC). In Section 6 we consider Beta processes, and use WH-MC to assess the accuracy of our approximation technique. In addition, as Beta processes are in $\mathscr{M}$, we can represent $\kappa(\alpha, q)$ as an infinite product; we also include the results obtained by truncating this product and performing the inversion.

\section{Preliminaries}

Recalling that we denote by $\bar{X}_{t}$ the running maximum process of the Lévy process $X_{t}$, and by $\tau(q)$ an exponentially distributed random variable (with mean $1 / q$, for $q>0$ ), we review in this section Lévy processes for which the double transform of $\bar{X}_{\tau(q)}$, denoted by $\kappa(\alpha, q)$, can be explicitly expressed in terms of the model's primitives, or immediately computable quantities.

We first consider the situation that there are no positive jumps, that is, the spectrally negative case. Following Bertoin (1998, Ch. VII), for $X \in \mathscr{S}_{-}$we define 
$\Phi(\beta):=\log \mathbb{E} e^{\beta X_{1}}$, and $\Psi(\cdot)$ its right-inverse (Kyprianou 2006, p. 211). Then $\kappa(\alpha, q)$ satisfies the following simple expression:

$$
\kappa(\alpha, q)=\frac{\Psi(q)}{\Psi(q)+\alpha} .
$$

In other words, $\bar{X}_{\tau(q)}$ is exponentially distributed with parameter $\Psi(q)$, or, equivalently,

$$
\int_{0}^{\infty} q e^{-q t} \mathbb{P}\left(\bar{X}_{t} \in \mathrm{d} x\right) \mathrm{d} t=\Psi(q) e^{-\Psi(q) x} \mathrm{~d} x
$$

Then we consider the case of no negative jumps, usually referred to as the spectrally positive case. For $X \in \mathscr{S}_{+}$we define the Laplace exponent by the function $\varphi(\cdot):[0, \infty) \mapsto[0, \infty)$, defined through $\varphi(\alpha):=\log \mathbb{E} e^{-\alpha X_{1}}$. In this case

$$
\kappa(\alpha, q)=\frac{q}{\psi(q)} \frac{\psi(q)-\alpha}{q-\varphi(\alpha)} .
$$

This result is sometimes referred to as the (generalized) Pollaczek-Khinchine formula (Harrison 1977; Zolotarev 1964); see also Asmussen (2003, Ch. IX, Thm. 3.10).

We finally consider the case in which the jumps in the downward direction are general, but those in the upward direction are assumed to have a rational Laplace transform (Lewis and Mordecki 2008). We define this class $\mathscr{R}$ by the Lévy processes $X_{t}$ such that for a finite and positive $\lambda$,

$$
\begin{aligned}
\xi(s):=\log \mathbb{E} e^{\mathrm{i} s X_{1}}= & \mathrm{i} s d-\frac{1}{2} s^{2} \sigma^{2}+\int_{-\infty}^{0}\left(e^{\mathrm{i} s x}-1-\mathrm{i} s x 1_{\{x>-1\}}\right) \Pi(\mathrm{d} x) \\
& +\lambda\left(\sum_{k=1}^{K} \sum_{j=1}^{n_{k}} c_{k j}\left(\frac{\mathrm{i} \alpha_{k}}{s+\mathrm{i} \alpha_{k}}\right)^{j}-1\right)
\end{aligned}
$$

where the $\alpha_{i}$ are order such that $0 \leq \operatorname{Re}\left(\alpha_{1}\right)<\operatorname{Re}\left(\alpha_{2}\right) \leq \cdots \leq \operatorname{Re}\left(\alpha_{K}\right)$. This corresponds to a Lévy process with a general jump-size distribution in the downwards direction, while the upwards jumps have density

$$
p(x)=\sum_{k=1}^{K} \sum_{j=1}^{n_{k}} c_{k j}\left(\alpha_{k}\right)^{j} \frac{x^{j-1}}{(j-1) !} e^{-\alpha_{k} x}, \quad x>0 .
$$

Now let $\beta_{j}(q)$ the $j$-th root of $q=\xi(s)$, with multiplicity $m_{j}(q)$; let $m(q)$ the total number of distinct roots. Then

$$
\kappa(\alpha, q)=\prod_{k=1}^{K}\left(\frac{\alpha+\alpha_{k}}{\alpha_{k}}\right)^{n_{k}} \prod_{j=1}^{m(q)}\left(\frac{\beta_{j}(q)}{\alpha+\beta_{j}(q)}\right)^{m_{j}(q)} ;
$$

this expression can be inverted with respect to $\alpha$, after having performed a partial fraction expansion. Further details and properties of the roots are given in Lewis and Mordecki (2008, Thm. 2.2). 


\section{Laplace Inversion}

As pointed out in the introduction, our approach requires a technique to perform Laplace transform inversion. More specifically, our methodology proposes a way to approximate the double transform $\kappa(\alpha, q)=\mathbb{E} e^{-\alpha \bar{X}_{\tau(q)}}$. In this section we first describe such a Laplace transform inversion technique in detail. As the objective of this section is to assess the accuracy of the double inversion technique, we then focus on a situation in which both $\kappa(\alpha, q)$ and $\mathbb{P}\left(\bar{X}_{t} \leq x\right)$ are explicitly known (viz. Brownian motion with drift). Then we consider situations for which we do know $\kappa(\alpha, q)$; for these cases we use simulation to validate our numerical findings.

\subsection{Laplace Inversion}

As indicated in the introduction, in our approach an important role is played by techniques to perform Laplace inversion. We advocate the use of the method developed by den Iseger (2006). It is in the spirit of approaches developed earlier (Abate and Whitt 1995; Dubner and Abate 1968), in the sense that it relies on the Poisson summation formula. This Poisson summation formula relates an infinite sum of Laplace transform values to the $z$-transform of the function values $f(k \Delta)$, with $k=0, \ldots, M-1$, that we wish to evaluate, from which the $f(k \Delta)$ can be computed relying on the well-known fast Fourier transform (Cooley and Tukey 1965).

A first complication is that the above-mentioned infinite sum tends to converge slowly. Abate and Whitt (1995) remedy this using a so-called Euler summation, but in general the convergence remains prohibitively slow unless knowledge of the location of singularities is available. One of den Iseger's contributions (den Iseger 2006) is to approximate the infinite sum by a finite sum by using a Gaussian quadrature. The resulting algorithm is a substantial improvement over earlier algorithms in the sense that (i) it can handle a larger class of Laplace transforms (e.g., no knowledge of the location of discontinuities or singularities is needed), (ii) the algorithm only needs numerical values of the Laplace transform, is fast (that is, the function values $f(k \Delta)$, with $k=0, \ldots, M-1$, are computed at once, in order $M \log M$ time), and is of nearly machine precision, (iii) can be extended to multiple dimensions. It is stressed that that last feature is of crucial importance to us, as in our setting we are often dealing with two-dimensional transforms.

In our numerical experiments we used the modified Laplace inversion for nonsmooth functions which was developed in den Iseger (2006, Section 6.2). This modification is effective for functions with discontinuities, singularities and local non-smoothness (even if we do not a priori know their locations). The experiments reported on in den Iseger (2006) show that the algorithm typically results in approximations of (nearly) machine precision. Below we explain in greater detail how this modification works.

Let $\hat{f}(s)$ be the Laplace transform of the complex-valued Lebesgue integrable function $f(x)$. Then it holds that (see e.g. Abate and Whitt 1995)

$$
\sum_{k=-\infty}^{\infty} \hat{f}(a+2 \pi \mathrm{i}(k+v))=\sum_{k=0}^{\infty} e^{-a k} e^{-2 \pi \mathrm{i} k v} f(k)
$$


where $a$ is a given real number. In this approach we approximate the left-hand side of Eq. 8 by a finite summation

$$
\sum_{k=1}^{n} \beta_{k} \hat{f}\left(a+\mathrm{i} \lambda_{k}+2 \pi \mathrm{i} v\right),
$$

where the $\left(\beta_{k}\right)_{k=1}^{n}$ are appropriately chosen positive numbers and $\left(\lambda_{k}\right)_{k=1}^{n}$ appropriately chosen real numbers. In den Iseger (2006, Appendix A) it is described how these numbers can be generated for such a quadrature rule.

Suppose now that $f(\cdot)$ has a singularity in $x=\alpha$ for some $\alpha \in \mathbb{R}$. Let $w(\cdot)$ be a window function, that is, a trigonometric polynomial with period 1 , with $w(0)=1$ and $w(\alpha)=0$. Define

$$
f_{w}(x):=w(x)^{q} f(x),
$$

for some positive integer $q$. The parameter $q$ is chosen such that $f_{w}(x)$ is smooth in $x=\alpha$; also observe that $f_{w}(k)$ coincides with $f(k)$ at $k=0,1, \ldots$ Now the 'normal' Laplace inversion technique, as described in den Iseger (2006, Section 4), applied to $\hat{f}_{w}(\cdot)$, can be used to compute the $f(k)$ (with integer $k$ ). If the function has multiple singularities, say in the points $\alpha_{j}$ with $j=1,2, \ldots, m$, the window function is the multiplication of window functions, that is, $w(x)=\prod_{j=1}^{m} w_{j}(x)$. If there is a singularity at $x=0$, a situation that occurs frequently in the examples of the present paper, the window function is

$$
w(x)=\sin ^{2}\left(\frac{\pi x}{2}\right)
$$

and in the way described above we can compute the function values $f(2 k+1)$. Guidelines for choosing the parameter $q$ are given in den Iseger (2006, Remark 6.5).

The modified algorithm described above can be improved for functions with various sorts of non-smoothness; we now describe an improvement detailed in den Iseger (2006, Section 6.3) which is useful when we do not know the location of the singularity. Suppose that the window function depends on the point $k, f_{w_{k}}(x)=$ $w_{k}(x) f(x)$, such that $w_{k}(k)=1$, and the $\varepsilon$-support, with $a>0$,

$$
\left\{x:\left|e^{-a t} f_{w_{k}}(x)\right| \geq \varepsilon\right\}
$$

of $f_{w_{k}}$ is $[k-\delta, k+\delta]$, with $\delta$ a given positive control parameter and $\varepsilon$ a predefined tolerance. In order to be sure that $f_{w_{k}}(\cdot)$ is smooth on $[0, \infty)$, it is sufficient that $f(\cdot)$ is smooth on $[k-\delta, k+\delta]$. As a result, it is only needed that $f(\cdot)$ be smooth on $[k-\delta, k+\delta]$ to compute $f(k)$ in great precision using the quadrature rule mentioned above. As it turns out, a good choice for the window function is the Gaussian function defined by

$$
w(t)=\exp \left(-\frac{1}{2}\left(\frac{t}{\sigma}\right)^{2}\right)
$$

for given tolerance $\epsilon$ and control parameter $\delta$, where $\sigma$ is chosen such that

$$
\exp \left(-\frac{1}{2}\left(\frac{\delta}{\sigma}\right)^{2}\right)<\epsilon .
$$


We also mention that den Iseger (2006, Section 5) points out how multi-dimensional inversion can be performed. For further implementation details we refer to den Iseger (2006).

This Laplace inversion method can be adjusted to facilitate the numerical computation of Laplace transforms; such a procedure is needed in situations that no explicit expressions are available (for instance for the Pareto or Weibull distribution). The key idea behind it concerns the transformation of the Legendre coefficients. Legendre polynomials are a complete orthogonal set of polynomials in $L^{2}([0,1])$ and, in addition, the shifted version of Legendre polynomials are a complete set in $L^{2}(\mathbb{R})$. Therefore, any function in $L^{2}(\mathbb{R})$ can be approximated with an expansion of shifted Legendre polynomials. On the other hand there is a complete set of functions in the Laplace domain; for a definition we refer to den Iseger and Oldenkamp (2006, Appendix A). The coefficients of the expansions in these two spaces are linked together through the Poisson summation formula (8). As demonstrated in den Iseger and Oldenkamp (2006), such a method can compute the Laplace transform with (almost) machine precision accuracy; it only needs knowledge of the coefficients of the expansion which can be computed by Gaussian quadratures.

In the rest of this section we systematically assess the performance of the inversion technique developed in den Iseger (2006) (and described above), in the context of the evaluation of $\mathbb{P}\left(\bar{X}_{t} \leq x\right)$. We start by considering a case in which explicit analysis is possible (viz. Brownian motion with drift). Then we consider a number of other examples for which no explicit expression is available (but in which we do know $\kappa(\alpha, q))$; in those cases we compare our numerical output with simulations.

\subsection{Comparison with Exact Results}

In this subsection we consider a case in which the distribution function of $\bar{X}_{t}$, that is, $\mathbb{P}\left(\bar{X}_{t} \leq x\right)$, is known explicitly.

Example 1 Let $X_{t}$ be a Brownian motion with drift, i.e., $X_{t}=d t+\sigma B_{t}$ with $B_{t}$ being standard Brownian motion and $d \in \mathbb{R}$. It holds that (Harrison 1985, p. 49)

$$
\mathbb{P}\left(\bar{X}_{t} \leq x\right)=1-\Phi_{\mathrm{N}}\left(\frac{-x+d t}{\sigma \sqrt{t}}\right)-e^{2 d x / \sigma^{2}} \Phi_{\mathrm{N}}\left(\frac{-x-d t}{\sigma \sqrt{t}}\right)
$$

with $\Phi_{\mathrm{N}}(\cdot)$ denoting the distribution function of a standard Normal random variable.

As highlighted in Section 3.1, several Laplace inversion variants are described in den Iseger (2006); they differ in the way they deal with discontinuities and singularities. In this example, and all following numerical computations presented in this paper, we use the variant described in den Iseger (2006, Section 6.3). Table 1 focuses on $\mathbb{P}\left(\bar{X}_{t} \leq x\right)$, and compares the output of our numerical experiments with the exact values and simulation-based estimates. Here, and in all other examples reported on in this paper, we perform $10^{7}$ independent replications per simulation experiment.

The third column contains the simulation-based estimate, the fourth the exact value based on the above formula. In the last two columns we use the explicit expression (4) that we have for $\kappa(\alpha, q)$ (or alternatively representation (6); recall that 
Table 1 Brownian motion with parameters $d=-0.5$ and $\sigma=1.0$

\begin{tabular}{llllll}
\hline Time $t$ & $x$ & Simulation & Exact value & Error, use Eq. 5 & Error, use Eq. 4 \\
\hline 0.1 & 0.1 & 0.286726 & 0.28679183 & $1.021 \mathrm{e}-14$ & $2.012 \mathrm{e}-11$ \\
& 0.2 & 0.525182 & 0.52535042 & $1.909 \mathrm{e}-14$ & $2.063 \mathrm{e}-11$ \\
& 0.5 & 0.912001 & 0.91208092 & $9.298 \mathrm{e}-15$ & $1.416 \mathrm{e}-10$ \\
0.3 & 1.0 & 0.999051 & 0.99906069 & $8.121 \mathrm{e}-17$ & $9.550 \mathrm{e}-10$ \\
& 0.1 & 0.190579 & 0.19063594 & $5.995 \mathrm{e}-15$ & $8.468 \mathrm{e}-11$ \\
& 0.2 & 0.358863 & 0.35900170 & $1.144 \mathrm{e}-14$ & $1.564 \mathrm{e}-10$ \\
& 0.5 & 0.723613 & 0.72378120 & $2.248 \mathrm{e}-14$ & $4.055 \mathrm{e}-10$ \\
0.5 & 1.0 & 0.959856 & 0.95991828 & $6.335 \mathrm{e}-15$ & $2.833 \mathrm{e}-09$ \\
& 0.1 & 0.161220 & 0.16126780 & $3.997 \mathrm{e}-15$ & $2.946 \mathrm{e}-10$ \\
& 0.2 & 0.305175 & 0.30529875 & $8.993 \mathrm{e}-15$ & $6.374 \mathrm{e}-10$ \\
& 0.5 & 0.636069 & 0.63611270 & $1.860 \mathrm{e}-14$ & $2.216 \mathrm{e}-09$ \\
& 1.0 & 0.908323 & 0.90832011 & $3.802 \mathrm{e}-15$ & $6.548 \mathrm{e}-09$ \\
\hline
\end{tabular}

Brownian motion is spectrally negative as well as spectrally positive!). In the fifth column we use the fact that we can perform the inversion with respect to $\alpha$ explicitly, as seen in Eq. 5; then a one-dimensional numerical Laplace inversion is used to approximate the probability of interest. The resulting error (compared to the exact result) is given. In the last column we present the values obtained when subjecting Eq. 4 to two-dimensional Laplace inversion; again the error is given. Observe that in the former approach error are maximally in the order of $10^{-14}$, and in the latter approach maximally of $10^{-9}$.

\subsection{Comparison with Simulation Results}

In the next set of examples, we let $X_{t}$ correspond to a Brownian motion with drift, plus a compound Poisson process with upward jumps. In other words,

$$
\log \mathbb{E} e^{\mathrm{i} s X_{1}}=\mathrm{i} s d-\frac{1}{2} s^{2} \sigma^{2}+\lambda\left(\mathbb{E} e^{\mathrm{i} s J}-1\right),
$$

with $J \geq 0$ the random variable associated with the jumps, and $\lambda>0$. As this process is spectrally positive, Eq. 6 applies. We consider various jump-size distributions $J$, thus covering both light-tailed and heavy-tailed scenarios.

One way to determine $\bar{X}_{t}$ in a simulation is by sampling the values of the Lévy process on a grid (yielding $X_{0}, X_{\Delta}, X_{2 \Delta}, \ldots, X_{t-\Delta}, X_{t}$ ), and to then take the maximum (tacitly assuming that $t$ is a multiple of $\Delta$ ). This procedure is inherently biased: the value found in this way is necessarily smaller than $\bar{X}_{t}$, but of course this bias decreases when $\Delta \downarrow 0$. In this section we consider the situation that the Lévy process is the sum of a deterministic drift, a Brownian term, and a compound Poisson process, and it turns out that for this specific scenario there is an attractive alternative. First observe that it is trivial to sample the jump epochs of the compound Poisson process up to time $t$, and the values of the Lévy process at these jump epochs, as well as the value at time $t$ itself; call the resulting time epochs $t_{0}=0, t_{1}, \ldots, t_{N-1}, t_{N}=t$. Then realize that the distribution of the maximum between $t_{i}$ and $t_{i+1}$ is knownit follows essentially from the distribution of the maximum attained by a Brownian bridge. The corresponding distribution function is invertible, and as a consequence it is elementary to sample from it. It is now clear that in this way we can generate all 
Table 2 Compound Poisson with exponential jumps

\begin{tabular}{llllll}
\hline Time $t$ & $x$ & Simulation & Appr., use Eq. 7 & Appr., use Eq. 6 & Difference \\
\hline 0.1 & 0.1 & 0.340062 & 0.34025703 & 0.34025703 & $1.32 \mathrm{e}-09$ \\
& 0.2 & 0.579295 & 0.57935467 & 0.57935467 & $1.24 \mathrm{e}-10$ \\
& 0.5 & 0.890919 & 0.89079051 & 0.89079051 & $2.04 \mathrm{e}-10$ \\
0.3 & 1.0 & 0.959618 & 0.95963405 & 0.95963405 & $4.18 \mathrm{e}-10$ \\
& 0.1 & 0.241525 & 0.24164945 & 0.24164945 & $5.67 \mathrm{e}-10$ \\
& 0.2 & 0.420271 & 0.42037262 & 0.42037262 & $9.24 \mathrm{e}-11$ \\
& 0.5 & 0.720007 & 0.71992788 & 0.71992788 & $4.34 \mathrm{e}-10$ \\
0.5 & 1.0 & 0.876619 & 0.87671756 & 0.87671756 & $3.04 \mathrm{e}-09$ \\
& 0.1 & 0.206808 & 0.20683652 & 0.20683652 & $4.94 \mathrm{e}-10$ \\
& 0.2 & 0.361211 & 0.36125826 & 0.36125826 & $7.48 \mathrm{e}-10$ \\
& 0.5 & 0.633832 & 0.63363062 & 0.63363062 & $1.57 \mathrm{e}-09$ \\
& 1.0 & 0.809454 & 0.80949895 & 0.80949895 & $1.16 \mathrm{e}-09$ \\
\hline
\end{tabular}

The jumps occur according to a Poisson process with rate $\lambda=1$; the jump sizes are exponential with mean 1. The Brownian term has parameters $d=-1.5$ and $\sigma=1.0$

information needed to determine $\bar{X}_{t}$ (without any approximation). The procedure is described in detail in Glynn and Mandjes (2011).

Example 2 In this example, we assume that jump size $J$ has an exponential distribution, that is, $\mathbb{P}(J>x)=\exp (-\mu x)$, with $\mu>0$. The results are presented in Table 2. Again, the third column contains the simulation-based estimate. In the fourth column, we rely on Eq. 7 with one-dimensional inversion (observe that the upward jumps are of phase-type, hence this formula applies); the resulting approximation is given. The fifth column displays the approximation based on Eq. 6 with two-dimensional inversion. Finally, the last column gives the difference between the previous two columns. It is concluded that both inversion-based methods are close to the simulation-based estimates; in addition, the inversion-based methods give nearly the same result (up to roughly $10^{-9}$, that is).

Table 3 Compound Poisson with Weibull jumps; heavy-tailed case

\begin{tabular}{llllll}
\hline Time $t$ & $x$ & Simulation & Appr., use Eq. 6 & Appr. Ph., use Eq. 7 & Difference \\
\hline 0.1 & 0.1 & 0.306441 & 0.30554528 & 0.30503331 & $5.12 \mathrm{e}-04$ \\
& 0.2 & 0.541505 & 0.53979428 & 0.53897538 & $8.19 \mathrm{e}-04$ \\
& 0.5 & 0.884350 & 0.88191833 & 0.88112773 & $7.90 \mathrm{e}-04$ \\
0.3 & 1.0 & 0.960646 & 0.95807537 & 0.95813022 & $5.48 \mathrm{e}-05$ \\
& 0.1 & 0.203235 & 0.20174542 & 0.20107108 & $6.74 \mathrm{e}-04$ \\
& 0.2 & 0.368249 & 0.36527241 & 0.36413314 & $1.14 \mathrm{e}-03$ \\
& 0.5 & 0.683732 & 0.67832431 & 0.67690345 & $1.42 \mathrm{e}-03$ \\
0.5 & 1.0 & 0.866122 & 0.85926538 & 0.85922344 & $4.19 \mathrm{e}-05$ \\
& 0.1 & 0.166972 & 0.16488486 & 0.16421784 & $6.67 \mathrm{e}-04$ \\
& 0.2 & 0.303935 & 0.29990763 & 0.29877142 & $1.13 \mathrm{e}-03$ \\
& 0.5 & 0.580408 & 0.57275238 & 0.57129482 & $1.46 \mathrm{e}-03$ \\
& 1.0 & 0.780043 & 0.76979656 & 0.76990473 & $1.08 \mathrm{e}-04$ \\
\hline
\end{tabular}

The jumps occur according to a Poisson process with rate $\lambda=1$; the jump sizes are Weibull with parameters $\mu=1.0$ and $\gamma=0.5$. The Brownian term has parameters $d=-1.0$ and $\sigma=1.0$. Number of Erlang distributions which were fitted to the Weibull distribution $N_{\mathrm{Er}}=7$ and the highest number of phases $n_{\max }=3$ 
Table 4 Compound Poisson with Weibull jumps; light-tailed case

\begin{tabular}{llllll}
\hline Time $t$ & $x$ & Simulation & Appr., use Eq. 6 ) & Appr. Ph., use Eq. 7 & Difference \\
\hline 0.1 & 0.1 & 0.298493 & 0.29822869 & 0.29822927 & $5.75 \mathrm{e}-07$ \\
& 0.2 & 0.526594 & 0.52650719 & 0.52650797 & $7.80 \mathrm{e}-07$ \\
& 0.5 & 0.861135 & 0.86135242 & 0.86133659 & $1.58 \mathrm{e}-05$ \\
0.3 & 1.0 & 0.953920 & 0.95401102 & 0.95437502 & $3.64 \mathrm{e}-04$ \\
& 0.1 & 0.189862 & 0.18976048 & 0.18978895 & $2.84 \mathrm{e}-05$ \\
& 0.2 & 0.343766 & 0.34370972 & 0.34376675 & $5.70 \mathrm{e}-05$ \\
& 0.5 & 0.643406 & 0.64373428 & 0.64389320 & $1.59 \mathrm{e}-04$ \\
0.5 & 1.0 & 0.848913 & 0.84918147 & 0.84978994 & $6.08 \mathrm{e}-04$ \\
& 0.1 & 0.152512 & 0.15236195 & 0.15243569 & $7.37 \mathrm{e}-05$ \\
& 0.2 & 0.277445 & 0.27746044 & 0.27759827 & $1.38 \mathrm{e}-04$ \\
& 0.5 & 0.536884 & 0.53709063 & 0.53740254 & $3.12 \mathrm{e}-04$ \\
& 1.0 & 0.761019 & 0.76122687 & 0.76199729 & $7.70 \mathrm{e}-04$ \\
\hline
\end{tabular}

The jumps occur according to a Poisson process with rate $\lambda=1$; the jump sizes are Weibull with parameters $\mu=1.0$ and $\gamma=2$. The Brownian term has parameters $d=-1.0$ and $\sigma=1.0$. Number of Erlang distributions which were fitted to the Weibull distribution $N_{\mathrm{Er}}=4$ and the highest number of phases $n_{\max }=5$

Example 3 Now let $J$ have a Weibull distribution: $\mathbb{P}(J>x)=\exp \left(-\mu x^{\gamma}\right)$, with $\mu, \gamma>0$. For $\gamma \in(0,1)$, this tail is heavier than exponential, for $\gamma>1$ lighter. More specifically, for $\gamma<1$ the Weibull distribution is subexponential: despite the fact that all moment exist, there is no open neighborhood around the origin such that the moment generating function is finite; in Table 3 the jump sizes are subexponential. The third column contains simulation-based estimates, the fourth is based on doubly inverting expression (6). Notice that in this situation we cannot approximate the probability $\mathbb{P}\left(\bar{X}_{t} \leq x\right)$ relying on Eq. 7 , as the positive jumps do not have a rational Laplace transform. The last two columns will be commented on in the next section. We observe that the approximation based on double Laplace inversion of Eq. 6 performs reasonably well compared to the simulation-based estimates; the fit is better in the light-tailed case.

Example 4 Let $J$ now be sampled from a Pareto distribution: $\mathbb{P}(J>x)=(x+1)^{-\gamma}$, for some $\gamma>0$. This tail is heavier than the Weibull-tail: just a finite number of moments exists-more precisely: the $k$-th moment exists if $k<\gamma$. Table 5 should be read as Tables 3 and 4 . We conclude that there is a good fit relative to the simulationbased results.

\section{Approximation Using Positive Jumps with Rational Laplace Transform}

From the examples presented in previous section we conclude that the numerical inversion procedure works well, even if the approximation requires a double inversion. In all these examples, however, the Lévy process involved was such that the double transform $\kappa(\alpha, q)$ was given in closed form.

In this section we add a complication. We consider cases in which $X_{t}$ is such that we do not have an explicit expression for $\kappa(\alpha, q)$. The focus will now be on Lévy processes that are Brownian motion with drift, plus compound Poisson processes (with upward and downward jumps); 'small jumps' will only be incorporated in the 
next section. If the jumps in the upward direction do not have a rational Laplace transform, the results of Lewis and Mordecki (2005) do not apply (see Section 2), and hence we do not have an explicit expression for $\kappa(\alpha, q)$. The idea is now that we approximate the distribution of the upward jumps by a phase-type distribution (while leaving the jumps in the downward direction unchanged), so that we are again in the framework of Lewis and Mordecki (2005)—realize that the class of phasetype distributions is contained in the class of distributions with a rational Laplace transform. The objective of this section is to assess how well such an approximation performs, in terms of evaluating $\mathbb{P}\left(\bar{X}_{t} \leq x\right)$.

\subsection{Fitting of Phase-Type Distributions}

There are various papers dealing with approximating a distribution on $(0, \infty)$ by a phase-type distribution, see for instance Feldmann and Whitt (1998) and Horváth and Telek (2324). In our work we rely on the approach developed in Asmussen et al. (1996), based on the EM algorithm, and Thümmler et al. (2006), who propose a comparable approach that focuses primarily on mixtures of Erlangs. For a precise definition of phase-type distributions, see e.g. Asmussen (2003, Ch. III); they can be thought of as distributions of absorption times in a finite-state continuoustime Markov chain. More precisely, with $d$ denoting the dimension of the state space, and $d-1$ states being transient and the remaining state absorbing, a phasetype distribution corresponds to the entrance time of the absorbing state. This class covers mixtures and sums of exponential distributions (and hence also the Erlang distribution, being distributed as the sum of independent exponential random variables with the same mean). The class of phase-type distributions is dense, in that any distribution on $(0, \infty)$ can, in principle, be approximated arbitrarily well; the price to be paid, though, is that the dimension $d$ of the associated Markov chain may become large.

The performance of the EM-based algorithm proposed is assessed in detail in Asmussen et al. (1996) - it was shown that quite a large class of distributions can be accurately approximated by phase-type distributions (of relatively low dimension $d$ ). From this it is, however, not a priori clear what the impact is of replacing the upward jumps by an appropriate phase-type random variable when evaluating $\mathbb{P}\left(\bar{X}_{t} \leq x\right)$ in the way described above-we do not have an explicit bound on the error introduced by replacing the jump distribution by its phase-type counterpart. It is the primary objective of this section to study this effect.

The remainder of this section consists of two parts. In Section 4.2 we consider models of which the upward jumps do not have a rational Laplace transform, but that are in $\mathscr{S}_{+}$(i.e., there are no downward jumps). Due to Eq. 6, we know $\kappa(\alpha, q)$, so that we can apply the inversion approach developed in den Iseger (2006) (see Section $3)$ to evaluate $\mathbb{P}\left(\bar{X}_{t} \leq x\right)$. Then we approximate the upward jumps with phasetype random variables, compute $\kappa(\alpha, q)$ relying on Lewis and Mordecki (2005), and again perform the inversion. Then we compare both numerical approximations of $\mathbb{P}\left(\bar{X}_{t} \leq x\right)$.

In Section 4.3 we consider models for which we do not know $\kappa(\alpha, q)$, i.e., models in which both the upward and downward jump have general distributions. We approximate the upward jumps by phase-type random variables, and proceed as before. We then compare with simulation to assess the accuracy of this approach. 


\subsection{Comparison with Results for Spectrally-Positive Lévy Processes}

In the examples below Eq. 9 applies: the Lévy process consists of a Brownian term (with drift) increased by a compound Poisson process with positive jumps. As a result, $\kappa(\alpha, q)$ is given by Eq. 6 . To assess the impact of replacing the upward jumps by their phase-type counterpart, we first use the EM-algorithm to find a phase-type approximation for the jumps, and then approximate $\mathbb{P}\left(\bar{X}_{t} \leq x\right)$, relying on Eq. 7 and a single-dimensional Laplace inversion.

Example 5 We go back to the setting of Example 3: we let $J$ have a Weibull distribution with $\gamma=0.5$ and $\gamma=2$, respectively. In the fifth column of Tables 3 and 4 we display the resulting numerical approximations. The last column gives the difference with the result of doubly inverting Eq. 6. It is concluded that the differences roughly range between $10^{-4}$ and $10^{-7}$.

Example 6 We now return to the setting of Example 4: we assume that $J$ corresponds to a Pareto distribution. The last two columns of Table 5 should be read as the corresponding columns in Tables 3 and 4 . Here the differences with the result based on Eq. 6 roughly range between $10^{-3}$ and $10^{-5}$.

Example 7 Now consider a slightly harder example: $J$ follows a shifted-Pareto distribution, that is, $\mathbb{P}(J>x)=1$ for $x \leq 1$, and $\mathbb{P}(J>x)=x^{-\gamma}$ for $x>1$, for some $\gamma>0$; observe that the support of $J$ is $(1, \infty)$. In this case the approximating phasetype distribution is a mixture of Erlang distributions of high degree; to this end, realize that an $\operatorname{Erl}(n, n)$ random variable (having mean 1 , and a variance $1 / n$, i.e., vanishing as $n$ grows large) approximates a deterministic(1) random variable. Table 6 should be read as Table 5. We observe that due the fact that the distribution of the jumps does not have support $(0, \infty)$, the phase-type-based approximation performs relatively weak.

Table 5 Compound Poisson with Pareto jumps

\begin{tabular}{llllll}
\hline Time $t$ & $x$ & Simulation & Appr., use Eq. 6 & Appr. Ph., use Eq. 7 & Difference \\
\hline 0.1 & 0.1 & 0.417032 & 0.41702642 & 0.41707991 & $5.35 \mathrm{e}-05$ \\
& 0.2 & 0.661758 & 0.66178212 & 0.66185155 & $6.94 \mathrm{e}-05$ \\
& 0.5 & 0.909879 & 0.91002954 & 0.91013778 & $1.08 \mathrm{e}-04$ \\
0.3 & 1.0 & 0.950149 & 0.95024208 & 0.95049174 & $2.50 \mathrm{e}-04$ \\
& 0.1 & 0.329487 & 0.32952345 & 0.32968987 & $1.66 \mathrm{e}-04$ \\
& 0.2 & 0.530314 & 0.53051325 & 0.53077411 & $2.61 \mathrm{e}-04$ \\
& 0.5 & 0.778595 & 0.77893652 & 0.77936986 & $4.33 \mathrm{e}-04$ \\
0.5 & 1.0 & 0.863911 & 0.86403154 & 0.86472833 & $6.97 \mathrm{e}-04$ \\
& 0.1 & 0.292768 & 0.29285157 & 0.29315023 & $2.99 \mathrm{e}-04$ \\
& 0.2 & 0.472333 & 0.47253618 & 0.47301425 & $4.78 \mathrm{e}-04$ \\
& 0.5 & 0.702192 & 0.70257211 & 0.70334963 & $7.77 \mathrm{e}-04$ \\
& 1.0 & 0.796445 & 0.79658771 & 0.79772318 & $1.13 \mathrm{e}-03$ \\
\hline
\end{tabular}

The jumps occur according to a Poisson process with rate $\lambda=1$; the jump sizes are Pareto with parameter $\gamma=1.0$. The Brownian term has parameters $d=-2.5$ and $\sigma=1.0$. Number of Erlang distributions which were fitted to the Pareto distribution $N_{\mathrm{Er}}=10$ and the highest number of phases $n_{\max }=5$ 
Table 6 Compound Poisson with shifted-Pareto jumps

\begin{tabular}{llllll}
\hline Time $t$ & $x$ & Simulation & Appr., use Eq. 6 & Appr. Ph., use Eq. 7 & Difference \\
\hline 0.1 & 0.1 & 0.409234 & 0.40893688 & 0.40897511 & $3.82 \mathrm{e}-05$ \\
& 0.2 & 0.647698 & 0.64752872 & 0.64761795 & $8.92 \mathrm{e}-05$ \\
& 0.5 & 0.881551 & 0.88133353 & 0.88190216 & $5.99 \mathrm{e}-04$ \\
0.3 & 1.0 & 0.912168 & 0.91201427 & 0.91587049 & $3.86 \mathrm{e}-03$ \\
& 0.1 & 0.302718 & 0.30249674 & 0.30373567 & $1.24 \mathrm{e}-03$ \\
& 0.2 & 0.485837 & 0.48568042 & 0.48783318 & $2.15 \mathrm{e}-03$ \\
& 0.5 & 0.704911 & 0.70473313 & 0.70947429 & $4.74 \mathrm{e}-03$ \\
0.5 & 1.0 & 0.779802 & 0.77970465 & 0.79028644 & $1.05 \mathrm{e}-02$ \\
& 0.1 & 0.252952 & 0.25275076 & 0.25585642 & $3.10 \mathrm{e}-03$ \\
& 0.2 & 0.407164 & 0.40696892 & 0.41214744 & $5.18 \mathrm{e}-03$ \\
& 0.5 & 0.600502 & 0.60036169 & 0.60967756 & $9.31 \mathrm{e}-03$ \\
& 1.0 & 0.685971 & 0.68588977 & 0.70143444 & $1.55 \mathrm{e}-02$ \\
\hline
\end{tabular}

The jumps occur according to a Poisson process with rate $\lambda=1$; the jump sizes are shifted-Pareto with parameter $\gamma=1.0$. The Brownian term has parameters $d=-2.5$ and $\sigma=1.0$. Number of Erlang distributions which were fitted to the shifted-Pareto distribution $N_{\mathrm{Er}}=12$ and the highest number of phases $n_{\max }=28$

\subsection{Comparison with Simulation Results}

In this subsection we deal with an example in which we do not know $\kappa(\alpha, q)$ (as opposed to the examples presented in Section 4.2).

Example 8 In this example we consider compound Poisson with two-sided jumps, plus Brownian motion with drift (Table 7). The upward jumps are Weibullian, and approximated by a phase-type distribution. The downward jumps are exponential. The numerical results are compared to simulation-based estimates, and show a good fit. (As an aside we mention that in this case the upward jumps are of phase-type,

Table 7 Compound Poisson with both upward and downward jumps

\begin{tabular}{llll}
\hline Time $t$ & $x$ & Simulation & Appr. Ph., use Eq. 7 \\
\hline 0.1 & 0.1 & 0.310605 & 0.31083581 \\
& 0.2 & 0.540572 & 0.54068394 \\
& 0.5 & 0.866999 & 0.86676851 \\
& 1.0 & 0.955574 & 0.95648076 \\
0.3 & 0.1 & 0.212655 & 0.21413828 \\
& 0.2 & 0.376741 & 0.37879054 \\
& 0.5 & 0.674504 & 0.67608908 \\
0.5 & 1.0 & 0.863495 & 0.86495277 \\
& 0.1 & 0.181433 & 0.19042446 \\
& 0.2 & 0.322121 & 0.33436792 \\
& 0.5 & 0.588677 & 0.59969483 \\
& 1.0 & 0.794101 & 0.80039848 \\
\hline
\end{tabular}

Both the upward jumps and downward jumps occur according to a Poisson process with rate $\lambda=1$; the positive jump sizes are Weibull with parameters $\mu=1$ and $\gamma=2$, and the negative jump sizes exponential with mean 1.0. The Brownian term has parameters $d=-1.0$ and $\sigma=1.0$. Number of Erlang distributions which were fitted to the Weibull distribution $N_{\mathrm{Er}}=5$ and the highest number of phases $n_{\max }=5$ 
where the downward jumps are not. Consequently, also in this case $\kappa(\alpha, q)$ can be given explicitly, in terms of a number of roots, see Lewis and Mordecki (2005). We do not pursue this approach.)

\section{Small Jumps}

So far we have developed a technique that can deal with all Lévy processes consisting of deterministic drifts, Brownian motions and compound Poisson processes. This means that we have not yet looked at processes with small jumps. In this section we rely on results from Asmussen and Rosiński (2004) to deal with these. The main result used is that under appropriate conditions a Lévy process with small jumps can be accurately approximated by the sum of an appropriately chosen compound Poisson process and Brownian motion. We first write the jump part of the Lévy exponent in the form

$\int_{-\infty}^{\infty}\left(e^{\mathrm{i} s x}-1-\mathrm{i} s x 1_{\{|x|<\varepsilon\}}\right) \Pi(\mathrm{d} x)=\int_{-\varepsilon}^{\varepsilon}\left(e^{\mathrm{i} s x}-1-\mathrm{i} s x\right) \Pi(\mathrm{d} x)+\int_{\mathbb{R} \backslash[-\varepsilon, \varepsilon]}\left(e^{\mathrm{i} s x}-1\right) \Pi(\mathrm{d} x) ;$

let the first term correspond to a Lévy process, say, $X_{t}^{(1, \varepsilon)}$, and the second term (which is a compound Poisson process) to, say, $X_{t}^{(2, \varepsilon)}$. Then the 'small jump component' $X_{t}^{(1, \varepsilon)}$ can be approximated by (for some small value of $\varepsilon$ )

$$
\mu_{\varepsilon} t+\sigma_{\varepsilon} B_{t}+X_{t}^{(2, \varepsilon)},
$$

where $B_{t}$ is a standard Brownian motion, and

$$
\mu_{\varepsilon}:=\int_{-\varepsilon}^{\varepsilon} x \Pi(\mathrm{d} x), \quad \sigma_{\varepsilon}^{2}:=\int_{-\varepsilon}^{\varepsilon} x^{2} \Pi(\mathrm{d} x)
$$

To shed some light on the accuracy of such an approximation, it is mentioned that it holds that under appropriate conditions (Asmussen and Rosiński 2004)

$$
\left(\frac{X^{(1, \varepsilon)}(t)-\mu_{\varepsilon} t}{\sigma_{\varepsilon}}\right)_{t \geq 0} \stackrel{\mathrm{d}}{\rightarrow}\left(B_{t}\right)_{t \geq 0}
$$

A sufficient condition for Eq. 11 to hold is that, with $L(\cdot)$ a slowly varying function at $0, \Pi(\cdot)$ has a density of the form $L(x) /|x|^{\gamma+1}$ for $x \downarrow 0$, with $\gamma \in(0,2)$. It is noted that this condition applies for e.g. stable Lévy processes and CGMY processes, but not for e.g. variance Gamma processes (as these correspond to $\gamma=0$ ). We also mention that the use of Eq. 10 is advocated for Variance Gamma in Fu (2007) — see his third algorithm on p. 25.

Approximating the distribution of the upward jumps by a phase-type distribution, we are again in the setting of Section 4. As a result, we can use the methodology developed earlier to perform the numerical computations. There is an obvious tradeoff between accuracy and computational effort when varying $\varepsilon$.

Example 9 In this example we consider a Lévy process whose upward jumps are CGMY-like, that is, for $C, M, Y>0$,

$$
\Pi(x)=C \frac{e^{-M x}}{x^{1+Y}}
$$


Table 8 The upward-jumps are CGMY-like, with parameters $C=1.0, M=2.0$ and $Y=0.5$; there are no downward jumps

\begin{tabular}{llllll}
\hline Time $t$ & $x$ & Simulation $\varepsilon=0.1$ & Simulation $\varepsilon=0.05$ & Appr., use Eq. 6 & Appr. Ph., use Eq. 7 \\
\hline 0.1 & 0.1 & 0.464093 & 0.464372 & 0.46468919 & 0.46685728 \\
& 0.2 & 0.707813 & 0.707969 & 0.70792216 & 0.71202807 \\
& 0.5 & 0.947668 & 0.947733 & 0.94756177 & 0.95056833 \\
0.3 & 1.0 & 0.993627 & 0.993675 & 0.99361188 & 0.99453926 \\
& 0.1 & 0.412429 & 0.412595 & 0.41282521 & 0.41675490 \\
& 0.2 & 0.636461 & 0.636570 & 0.63645668 & 0.64307083 \\
& 0.5 & 0.896372 & 0.896337 & 0.89611778 & 0.90206520 \\
0.5 & 1.0 & 0.981780 & 0.981841 & 0.98174473 & 0.98406940 \\
& 0.1 & 0.402468 & 0.402620 & 0.40282695 & 0.40744480 \\
& 0.2 & 0.621987 & 0.622003 & 0.62191009 & 0.62953509 \\
& 0.5 & 0.882154 & 0.882088 & 0.88182180 & 0.88897926 \\
& 1.0 & 0.976001 & 0.975993 & 0.97592972 & 0.97898461 \\
\hline
\end{tabular}

The Brownian term has parameters $d=-4.0$ and $\sigma=1.0$. Number of Erlang distributions which were fitted to the CGMY-upper tail $N_{\mathrm{Er}}=5$ and the highest number of phases $n_{\max }=9$, after having cut off the interval $(0, \varepsilon)$, with $\varepsilon=0.1$, from $\Pi(\cdot)$

for $x>0$ and 0 else. A Brownian term is added. The third and fourth column of Table 8 present simulation-based estimates, based on approximation (10), with $\varepsilon=$ 0.1 and $\varepsilon=0.05$, respectively. Observe that this model is contained in $\mathscr{S}_{+}$, so that Eq. 6 applies and $\kappa(\alpha, q)$ is given explicitly; the fifth column gives the results based on double inversion of Eq. 6. In the last column $X_{t}^{(2, \varepsilon)}$ is approximated by a Lévy process with phase-type jumps; as usual, we apply Eq. 7. From the small difference between both simulation-based columns, we conclude that those values are likely to be close to the true values. The inversion-based columns are well in agreement with each other and with the simulation-based output.

Example 10 We now consider a Variance Gamma process, which a can be regarded as a (standard, in our case) Brownian motion where the time parameter follows a Gamma process. More precisely, with $B_{t}$ being a standard Brownian motion, and $Y_{t}$ a Gamma process with parameters 1 and 1, the Lévy process under consideration is given by $B_{Y_{t}}$; this is an example of subordinated Brownian motion.

The third column of Table 9 presents simulation-based estimates, based on approximation (10), with $\varepsilon=0.01$. The fourth column also gives simulation-based estimates, but now simulating the Variance Gamma process as subordinated Brownian motion. This means that we sample the values of the Gamma process on a grid, and then generate the Brownian motion at these values. We have performed this procedure for different grid sizes, $N=200, N=500, N=1000$ but we observed just minor differences (negligible with respect to the width of the confidence interval).

To obtain the fifth column, the upper tail of the Lévy measure is split into a Brownian component and a compound Poisson component, as explained earlier in this section. We observe a reasonable fit. The problem with the approach that we proposed, however, is that we cut out the interval $(0, \varepsilon)$, so that the positive jumps have a distribution which has support $(\varepsilon, \infty)$-we saw before (viz. in the shiftedPareto case) that such distributions do not lend themselves to be approximated by a phase-type distribution. We can remedy this effect by allowing the jump size distribution to have support $(0, \infty)$; we give the Lévy measure of $X_{t}^{(2, \varepsilon)}$ the value 
Table 9 Variance Gamma process, $d=0.0, \sigma^{2}=1.0$ and $\kappa=1.0$

\begin{tabular}{llllll}
\hline Time $t$ & $x$ & Simulation $\varepsilon=0.01$ & Simulation SBM & Appr. Ph., use Eq. 7 & Appr. Ph. adapted \\
\hline 0.1 & 0.1 & 0.862579 & 0.863298 & 0.87678746 & 0.85836619 \\
& 0.2 & 0.909530 & 0.909840 & 0.92167074 & 0.90125495 \\
& 0.5 & 0.962386 & 0.962496 & 0.96661308 & 0.96199422 \\
0.3 & 1.0 & 0.987754 & 0.987790 & 0.98923639 & 0.98548799 \\
& 0.1 & 0.663389 & 0.665469 & 0.68917587 & 0.65958697 \\
& 0.2 & 0.759177 & 0.760215 & 0.78453098 & 0.74556038 \\
& 0.5 & 0.886828 & 0.887072 & 0.89861106 & 0.88560877 \\
0.5 & 1.0 & 0.959078 & 0.959243 & 0.96420072 & 0.95351051 \\
& 0.1 & 0.536040 & 0.538339 & 0.56558233 & 0.54403583 \\
& 0.2 & 0.646397 & 0.647893 & 0.67988532 & 0.64197788 \\
& 0.5 & 0.816052 & 0.816467 & 0.83582281 & 0.81968867 \\
& 1.0 & 0.927131 & 0.927450 & 0.93698719 & 0.92169160 \\
\hline
\end{tabular}

For the fifth column, number of Erlang distributions which were fitted to the upper tail $N_{\mathrm{Er}}=10$ and the highest number of phases $n_{\max }=10$, after having cut off the interval $(0, \varepsilon)$, with $\varepsilon=0.01$, from $\Pi(\cdot)$. For the last column, number of Erlang distributions which were fitted to the upper tail $N_{\mathrm{Er}}=3$ and the highest number of phases $n_{\max }=4$, after having cut off the interval $(0, \varepsilon)$, with $\varepsilon=0.01$, from $\Pi(\cdot)$

$\Pi(\varepsilon)$ in the interval $(0, \varepsilon)$; the parameters of the Brownian motion are then adapted such that the first two moments give the desired match. The last column gives the resulting estimates; the fit is considerable better than in the previous column and in addition the dimension of the approximating mixture of Erlangs is substantially lower.

\section{Beta Processes}

In this section we test our methodology for the class of Beta processes, which fall in the class of Lévy processes $\mathscr{M}$ for which the Lévy exponent is meromorphic. For these processes, $\kappa(\alpha, q)$ can be represented (Kuznetsov 2010) in terms of an infinite product. By truncating this infinite product and performing a one-dimensional inversion, we can approximate $\mathbb{P}\left(\bar{X}_{t} \leq x\right)$. We also obtain a simulation-based benchmark, by performing the sampling method developed by Kuznetsov et al. (2011). We start this section by reviewing this simulation technique.

\subsection{Wiener-Hopf Monte Carlo (WH-MC) Simulating Method}

Suppose that we are able to sample the running maximum $\left(\bar{X}_{\tau(q)}\right)$ and the running minimum $\left(\underline{X}_{\tau(q)}\right)$, where $\tau(q)$ is a exponentially distributed random variable with mean $1 / q$. Then by the method developed by Kuznetsov et al. (2011), based on an algorithm introduced by Carr (1998), we are able to evaluate $\mathbb{E}\left[F\left(X_{t}, \bar{X}_{t}\right)\right]$, the main ideas being the following. By the strong law of large numbers we know that $\sum_{i=1}^{n} \frac{t}{n} \boldsymbol{e}_{i} \rightarrow t$ as $n \rightarrow \infty$, where $\boldsymbol{e}_{i}$ constitutes a sequence of i.i.d. exponentially distributed random variables with mean 1 . The random variable $\sum_{i=1}^{n} \frac{t}{n} \boldsymbol{e}_{i}$ is equal in law to gamma random variable with parameters $n$ and $q=\frac{n}{t}$; we denote it by $g(n, q)$. As a consequence, $\mathbb{P}\left(X_{g(n, q)} \in \mathrm{d} x, \bar{X}_{g(n, q)} \in \mathrm{d} y\right)$ is a suitable approximation 
to $\mathbb{P}\left(X_{t} \in \mathrm{d} x, \bar{X}_{t} \in \mathrm{d} y\right)$, taking $n$ sufficiently large. The following result is due to Kuznetsov et al. (2011, Thm. 1), to which we refer for more details.

Theorem 1 For all $n \geq 1$ and $q>0$, define $g(n, q):=\sum_{i=1}^{n} \frac{t}{n} \boldsymbol{e}_{i}$. Then

$$
\mathbb{P}\left(X_{g(n, q)} \in \mathrm{d} x, \bar{X}_{g(n, q)} \in \mathrm{d} y\right) \stackrel{\mathrm{d}}{=}(V(n, q), J(n, q))
$$

where $V(n, q)$ and $J(n, q)$ are defined iteratively through

$$
\begin{aligned}
& V(n, q)=V(n-1, q)+S_{q}^{(n)}+I_{q}^{(n)} \\
& J(n, q)=\max \left(J(n-1, q), V(n-1, q)+S_{q}^{(n)}\right)
\end{aligned}
$$

and $V(0, q)=J(0, q)=0, S_{q}^{(0)}=I_{q}^{(0)}=0,\left\{S_{q}^{(j)} ; j \geq 1\right\}$ is a sequence of i.i.d. random variables with common distribution $\bar{X}_{\tau(q)}$, and $\left\{I_{q}^{(j)} ; j \geq 1\right\}$ is a sequence of i.i.d. random variables with common distribution $\underline{X}_{\tau(q)}$.

\subsection{Beta Processes}

The class of Beta processes consists of Lévy processes defined by the triplet $(\mu, \sigma, \Pi)$, where the Lévy measure is defined as

$$
\Pi(x)=c_{1} \frac{e^{-\alpha_{1} \beta_{1} x}}{\left(1-e^{-\beta_{1} x}\right)^{\lambda_{1}}} 1_{\{x>0\}}+c_{2} \frac{e^{\alpha_{2} \beta_{2} x}}{\left(1-e^{\beta_{2} x}\right)^{\lambda_{2}}} 1_{\{x<0\}},
$$

with parameters $\alpha_{i}>0, \beta_{i}>0, c_{i} \geq 0$ and $\lambda_{i} \in(0,3) \backslash\{1,2\}$. Its Lévy exponent is

$$
\Psi(s)=\mathrm{i}(\mu-\rho) s-\frac{1}{2} \sigma^{2} s^{2}+\frac{c_{1}}{\beta_{1}} \mathbf{B}\left(\alpha_{1}-\frac{\mathrm{i} s}{\beta_{1}}, 1-\lambda_{1}\right)+\frac{c_{2}}{\beta_{2}} \mathbf{B}\left(\alpha_{2}+\frac{\mathrm{i} s}{\beta_{2}}, 1-\lambda_{2}\right)-\gamma
$$

Here $\mathbf{B}(x, y):=\Gamma(x) \Gamma(y) / \Gamma(x+y)$ is the well-known Beta function. In addition, with $\psi(x):=\mathrm{d} \log (\Gamma(x)) / \mathrm{d} x$,

$$
\begin{aligned}
\gamma= & \frac{c_{1}}{\beta_{1}} \mathbf{B}\left(\alpha_{1}, 1-\lambda_{1}\right)+\frac{c_{2}}{\beta_{2}} \mathbf{B}\left(\alpha_{2}, 1-\lambda_{2}\right), \\
\rho= & \frac{c_{1}}{\beta_{1}} \mathbf{B}\left(\alpha_{1}, 1-\lambda_{1}\right)\left(\psi\left(1+\alpha_{1}-\lambda_{1}\right)-\psi\left(\alpha_{1}\right)\right) \\
& -\frac{c_{2}}{\beta_{2}} \mathbf{B}\left(\alpha_{2}, 1-\lambda_{2}\right)\left(\psi\left(1+\alpha_{2}-\lambda_{2}\right)-\psi\left(\alpha_{2}\right)\right) .
\end{aligned}
$$

The Lévy exponent of the beta process is a meromorphic function in $\mathbb{C}$; it turns out to be possible to identify all roots of the equation $q-\Psi(s)=0$; these roots are characterized in the following theorem (Kuznetsov 2010, Thm. 10). 
Theorem 2 For $q>0$ and $\Psi(s)$ defined above, the equation $q-\Psi(\mathrm{i} \xi)=0$ has infinitely many solutions, all of which are real and simple. they are such that $\xi_{0}^{-} \in$ $\left(-\alpha_{1} \beta_{1}, 0\right)$ and $\xi_{0}^{+} \in\left(0, \alpha_{2} \beta_{2}\right)$, while for $n \in\{1,2, \ldots\}$,

$$
\xi_{n}^{-} \in\left(\beta_{1}\left(-\alpha_{1}-n\right), \beta_{1}\left(-\alpha_{1}-n+1\right)\right), \quad \xi_{n}^{+} \in\left(\beta_{2}\left(\alpha_{2}+n-1\right), \beta_{2}\left(\alpha_{2}+n\right)\right) .
$$

Moreover, for $x>0$,

$$
\mathbb{P}\left(\bar{X}_{\tau(q)} \in \mathrm{d} x\right)=-\left(\sum_{k=0}^{\infty} C_{k}^{-} \xi_{k}^{-} e^{\xi_{k}^{-} x}\right) \mathrm{d} x
$$

where, with $k \in\{1,2, \ldots\}$,

$$
\begin{aligned}
C_{0}^{-} & =\prod_{n \geq 1} \frac{1+\xi_{0}^{-} / \beta_{1}\left(n-1+\alpha_{1}\right)}{1-\xi_{0}^{-} / \xi_{n}^{-}}, \\
C_{k}^{-} & =\frac{1+\xi_{k}^{-} / \beta_{1}\left(k-1+\alpha_{1}\right)}{1-\xi_{k}^{-} / \xi_{0}^{-}} \prod_{n \geq 1, n \neq k} \frac{1+\xi_{k}^{-} / \beta_{1}\left(n-1+\alpha_{1}\right)}{1-\xi_{k}^{-} / \xi_{n}^{-}} .
\end{aligned}
$$

A similar expression holds for $\mathbb{P}\left(-\underline{X}_{\tau(q)} \in \mathrm{d} x\right)$, but $\left\{\xi_{n}^{-}\right\}$must be replaced by $\left\{-\xi_{n}^{+}\right\}$ and $\alpha_{1}, \beta_{1}$ must be replaced by $\alpha_{2}, \beta_{2}$.

Note that if $\sigma=0$ and $\lambda_{i}<2$ the distribution of $\bar{X}_{\tau(q)}$ has an atom at zero which is equal to $1-\sum_{n \geq 1} C_{k}^{-}$; it can be written as $\prod_{n \geq 0}-\xi_{n}^{-} / \beta_{1}\left(n+\alpha_{1}\right)$.

The above theorem provides us with information about the location of the poles, thus facilitating the efficient determination of their exact positions (use for instance a simple bisection method). However, for performing the inverse Laplace transform we need to find poles for complex values of $q$; as computing roots for complex $q$ is time consuming, we rely on the method that we explain in Section 7.2.

Example 11 In this example, we consider a Beta process with parameters

$$
\left(\mu, \sigma ; \alpha_{1}, \beta_{1}, \lambda_{1}, c_{1} ; \alpha_{2}, \beta_{2}, \lambda_{2}, c_{2}\right)=(-0.5,1 ; 1,1.5,1.5,1 ; 1,1.5,1.5,1) .
$$

Table 10 Beta process, with parameters of Example 11

\begin{tabular}{llllllll}
\hline $\begin{array}{l}\text { Time } \\
t\end{array}$ & $x$ & $\begin{array}{l}\text { Simulation } \\
\varepsilon=0.1\end{array}$ & $\begin{array}{l}\text { Simulation } \\
\varepsilon=0.05\end{array}$ & $\begin{array}{l}\text { WH-MC } \\
n=20\end{array}$ & $\begin{array}{l}\text { WH-MC } \\
n=100\end{array}$ & $\begin{array}{l}\text { Laplace } \\
\text { inversion (14) }\end{array}$ & $\begin{array}{l}\text { Appr. Ph. } \\
\text { adapted }\end{array}$ \\
\hline 0.1 & 0.1 & 0.2693 & 0.2696 & 0.2760 & 0.2713 & 0.2696 & 0.2626 \\
& 0.2 & 0.4849 & 0.4850 & 0.4961 & 0.4883 & 0.4850 & 0.4707 \\
& 0.5 & 0.8476 & 0.8474 & 0.8517 & 0.8475 & 0.8475 & 0.8367 \\
& 1.0 & 0.9710 & 0.9711 & 0.9686 & 0.9675 & 0.9710 & 0.9708 \\
0.3 & 0.1 & 0.1724 & 0.1726 & 0.1761 & 0.1732 & 0.1725 & 0.1788 \\
& 0.2 & 0.3162 & 0.3163 & 0.3235 & 0.3185 & 0.3163 & 0.3213 \\
& 0.5 & 0.6255 & 0.6255 & 0.6359 & 0.6287 & 0.6255 & 0.6187 \\
0.5 & 1.0 & 0.8703 & 0.8702 & 0.8714 & 0.8688 & 0.8703 & 0.8605 \\
& 0.1 & 0.1421 & 0.1423 & 0.1450 & 0.1425 & 0.1422 & 0.1580 \\
& 0.2 & 0.2613 & 0.2615 & 0.2669 & 0.2625 & 0.2613 & 0.2822 \\
& 0.5 & 0.5291 & 0.5290 & 0.5389 & 0.5316 & 0.5291 & 0.5427 \\
\hline
\end{tabular}

Number of Erlang distributions which were fitted to the upper tail $N_{\mathrm{Er}}=3$ and the highest number of phases $n_{\max }=4$, after having cut off the interval $(0, \varepsilon)$, with $\varepsilon=0.1$, from $\Pi(\cdot)$ 
Because the Beta process is a Lévy process with small jumps, we need to approximate the jumps smaller than $\varepsilon$ with a Brownian motion in the Monte-Carlo simulation; in the Wiener-Hopf Monte-Carlo simulation we do not need this approximation. It is also noted that the distributions of $\bar{X}_{\tau(q)}$ and $\underline{X}_{\tau(q)}$ are expressed in terms of infinite series, and as a consequence we have to perform a truncation to sample from $\bar{X}_{\tau(q)}$ and $\underline{X}_{\tau(q)}$. In Table 10 the third and forth columns show the result obtained from 'ordinary simulation', using $\varepsilon=0.1$ and $\varepsilon=0.05$. The next two columns display the estimates obtained relying on WH-MC with the number of iterations $n$ in Theorem 2 equal to 20 and 100, respectively. We perform $10^{7}$ realizations in each simulation.

The seventh column shows the outcome of Eq. 14, where the summation is truncated after 25 terms. The last column, finally, is based on inverting the Laplace transform obtained by approximating the positive jumps by their phase-type counterparts. If we leave out the jumps smaller than $\varepsilon$ the positive jumps size distribution will have support $(\varepsilon, \infty)$ which is poorly approximated by Erlang distributions, as we explained in Example 10; we remedy this complication in the same way as we did in Example 10.

\section{Discussion and Concluding Remarks}

We conclude our paper by briefly discussing a number of issues that affect the accuracy and computation time.

\subsection{Remarks on Fitting of Phase-Type Distribution}

Let $f(x)$ be the density which we wish to approximate with a so-called hyper-Erlang distribution of degree $N$, that is, we wish to find $\alpha_{j}, \lambda_{j}$ and $n_{j}$ such that

$$
f(x) \approx \sum_{j=1}^{N} \alpha_{j} \frac{\left(\lambda_{i} x\right)^{n_{j}-1}}{\left(n_{j}-1\right) !} \lambda_{j} e^{-\lambda_{j} x} ;
$$

here $n_{1}, \ldots, n_{N} \in \mathbb{N}$ are the numbers of phases of the individual Erlang distributions, the $\lambda_{j}$ s are positive numbers, while the $\alpha_{j}$ s are positive numbers such that $\sum_{j=1}^{N} \alpha_{j}=1$.

With the EM algorithm we can optimize the parameters $\alpha_{j}$ and $\lambda_{j}$ for a given $N$ and predefined set of $n_{j}$. In order to find the 'best' fitting we have tried a large set of vectors $\left(n_{1}, n_{2}, \cdots, n_{N}\right)$ for a given $N$, in order to identify the $\left(n_{1}, n_{2}, \cdots, n_{N}\right)$ which maximizes the likelihood. This procedure can be implemented efficiently; for a detailed discussion we refer to Thümmler et al. (2006).

Note that all phase-type distributions have the features that they (i) are lighttailed, and (ii) have support $(0, \infty)$. As a consequence, it heavily depends on the distribution under consideration whether it can be approximated well by a phase-type distribution. From our experiments, we observed that for light-tailed distributions Erlang distributions of low dimension suffice; see for example the Weibull distribution with $\gamma \geq 1$. Distributions with heavier tails (Pareto, the Weibull distribution with $\gamma<1$ ) are significantly harder to approximate (in that they require a mixture of Erlangs of high dimension); it is emphasized that the fit of the distribution's tail may be poor in this case, while the 'body' of the distribution is approximated quite 
well. Distributions with support different from $(0, \infty)$ are even harder to fit; think of the shifted-Pareto distribution. In this case, recall that the approximating phasetype distribution contains multiple Erlang distributions of high degree; note that an $\operatorname{Erl}(n, n)$ random variable (which has mean 1 , and variance $1 / n$, i.e., vanishing as $n$ grows large) can be used to approximate a deterministic(1) random variable. Our experiments indicate that, despite the fact that we included such high-degree Erlang distributions, the resulting numerics are decent, but not highly accurate.

\subsection{Remarks on the Computation Time}

As we mentioned earlier in the paper, the computation time of the Laplace inversion algorithm is of the order $M \log (M)$, if function values $f(k \Delta), k=0,1, \cdots, M-1$ are to be computed. It is emphasized, though, that the bulk of the computation time is not related to this inversion, but rather to the numerics related to identifying the roots $\beta_{j}(q)$ in Eq. 7, which solve $q=\xi(s)$. The number of roots of this equation equals to $N_{\mathrm{p}}+1$ if there is a Brownian component in the Lévy exponent, and $N_{\mathrm{p}}$ otherwise; here $N_{\mathrm{p}}$ denotes the sum of phases of the individual distributions the phase-type distribution is composed from. For details we refer to Lewis and Mordecki (2005).

In general, finding these roots can be extremely time consuming, as we lack precise knowledge about the locations of the roots in the complex plane and their multiplicity. In addition, the Laplace inversion algorithm needs to compute these roots for different values of $q$. In order to save time, we first compute the roots $\beta_{j}$ of the equation $a=\xi(s)$ with $a$ being real damping factor. Note that the roots of the equation $a+\mathrm{i} q=\xi(s)$ change continuously with respect to $q$ in the complex plane; considering the roots as explicit functions of $q$ such that

$$
\xi\left[\beta_{j}(q)\right]=a+\mathrm{i} q ; \quad \beta_{j}(0)=\beta_{j}
$$

we obtain by differentiating with respect to $q$ the ordinary differential equation

$$
\frac{\mathrm{d} \beta_{j}(q)}{\mathrm{d} q}=\frac{\mathrm{i}}{\xi^{\prime}\left[\beta_{j}(q)\right]} .
$$

Applying this procedure, we can find the roots efficiently for different values of $q$ by using, for example, an adaptive Runge-Kutta method (Kuznetsov 2010).

Acknowledgment We thank Martijn Pistorius for carefully reading the manuscript, and for providing useful suggestions.

Open Access This article is distributed under the terms of the Creative Commons Attribution License which permits any use, distribution, and reproduction in any medium, provided the original author(s) and the source are credited.

\section{References}

Abate J, Whitt W (1995) Numerical inversion of Laplace transforms of probability distributions. ORSA J Comput 7:36-43

Asmussen S (2003) Applied probability and queues. Springer, New York

Asmussen S, Rosiński J (2004) Approximations of small jumps of a Lévy process with a view towards simulation. J Appl Probab 38:482-493 
Asmussen S, Nerman O, Olsson M (1996) Fitting phase-type distributions via the EM algorithm. Scand J Statist 23:419-441

Asmussen S, Avram F, Pistorius M (2004) Russian and American put options under exponential phase-type Lévy models. Stoch Process Their Appl 109:79-111

Asmussen S, Madan D, Pistorius M (2007) Pricing equity default swaps under an approximation to the CGMY Lévy model. J Comput Financ 11:79-93

Bertoin J (1998) Lévy processes. Cambridge University Press, Cambridge

Carr P (1998) Randomization and the American Put. Rev Financ Stud 11:597-626

Cooley J, Tukey J (1965) An algorithm for the machine calculation of complex Fourier series. Math Comput 19:297-301

Cont R, Tankov P (2004) Financial modelling with Jump processes. Chapman \& Hall/CRC Press, Boca Raton

den Iseger P (2006) Numerical transform inversion using Gaussian quadrature. Probab Eng Inf Sci 20:1-44

den Iseger P, Oldenkamp E (2006) Pricing guaranteed return rate products and discretely sampled Asian options. J Comput Financ 9:383-403

Dębicki K, Mandjes M (2012) Lévy-driven queues. Surv Oper Res Manage Sci 17:15-37

Dubner H, Abate J (1968) Numerical inversion of Laplace transforms by relating them to the finite Fourier cosine transform. J ACM 15:115-123

Feldmann A, Whitt W (1998) Fitting mixtures of exponentials to long-tail distributions to analyze network performance models. Perform Eval 31:245-279

Fu M (2007) Variance-Gamma and Monte Carlo. In: Fu J, Yen E (eds) Advances in mathematical finance. Birkhäuser, Basel, pp 21-35

Glynn P, Mandjes M (2011) Simulation-based computation of workload correlation function in a Levy-driven queue. J Appl Probab 48(1):114-130

Harrison J (1977) The supremum distribution of a Lévy process with no negative jumps. Adv Appl Probab 9:417-422

Harrison J (1985) Brownian motion and stochastic flow systems. Wiley, New York

Horváth A, Telek M (2002) Phfit: a general phase-type fitting tool. In: Proc. of 12th performance TOOLS, LNCS 2324, pp 82-91

Kuznetsov A (2010) Wiener-Hopf factorization and distribution of extrema for a family of Lévy processes. Ann Appl Probab 20:1801-1830

Kuznetsov A, Kyprianou A, Pardo J, van Schaik K (2011) A Wiener-Hopf Monte Carlo simulation technique for Lévy processes. Ann Appl Probab 21:2171-2190

Kyprianou A (2006) Introductory lectures on fluctuations of Lévy processes with applications. Springer, Berlin

Lewis A, Mordecki E (2005) Wiener-Hopf factorization for Lévy processes having negative jumps with rational transforms. Submitted for publication

Lewis A, Mordecki E (2008) Wiener-Hopf factorization for Lévy processes having positive jumps with rational transforms. J Appl Probab 45:118-134

Prabhu N (1998) Stochastic storage processes, 2nd edn. Springer, New York

Rogers L (2000) Evaluating first-passage probabilities for spectrally one-sided Lévy processes. J Appl Probab 37:1173-1180

Surya B (2008) Evaluating scale functions of spectrally negative Lévy processes. J Appl Probab 45:135-149

Thümmler A, Buchholz P, Telek M (2006) A novel approach for phase-type fitting with the EM Algorithm. IEEE Trans Dep Sec Comp 3:245-258

Zolotarev V (1964) The first passage time of a level and the behaviour at infinity for a class of processes with independent increments. Theory Probab Appl 9:653-661 